\title{
THE DISTRIBUTION OF ZEROS OF SOLUTIONS OF FIRST ORDER FUNCTIONAL DIFFERENTIAL EQUATIONS
}

\author{
YONG ZHOU
}

In this paper, we establish some estimates for the distance between adjacent zeros of the solutions of the first order delay differential equation

$$
x^{\prime}(t)+P(t) x(t-\tau)=0
$$

and the first order advanced differential equation

$$
x^{\prime}(t)-P(t) x(t+\tau)=0
$$

where $P \in C\left(\left[t_{0}, \infty\right),[0, \infty)\right)$ and $r \in R^{+}$. Our results improve recent results in the literature.

\section{INTRODUCTION}

The oscillation theory of delay differential equations has been extensively developed during the past several years. We refer to the monographs by Erbe, Kong and Zhang [1], Gopalsamy [2], Györi and Ladas [3] and the references cited therein. But results dealing with the distribution of zeros of the oscillatory solutions of delay differential equations are relatively scarce. Erbe et al. [3], Liang [4], and Zhou [5] established estimates for the distance between adjacent zeros of the solutions of first order delay differential equations. Recently, Zhou $[6,7,8]$ and Wang $[8]$ studied the distribution of zeros of the oscillatory solutions of neutral differential equations.

In this paper, we consider first order delay differential equations of the form

$$
x^{\prime}(t)+P(t) x(t-\tau)=0,
$$

and first order advanced differential equations of the form

$$
x^{\prime}(t)-P(t) x(t+\tau)=0,
$$

where

$$
P \in C\left(\left[t_{0}, \infty\right),[0, \infty)\right), \tau>0 .
$$

By using a new technique, we obtain a better estimate for the distance between adjacent zeros of the solutions of $(1)$ which improve the results in $[1,4,5]$. We also extend this result to (2).

Received 2nd September, 1998

This research was supported by the National Natural Science Foundation of the People's Republic of China.

Copyright Clearance Centre, Inc. Serial-fee code: 0004-9729/99 \$A2.00+0.00. 


\section{LEMMAS}

First we define a sequence $\left\{f_{n}(\rho)\right\}$ by

$$
f_{1}(\rho)=e^{\rho}, f_{n+1}(\rho)=e^{\rho f_{n}(\rho)}, n=1,2, \ldots
$$

where $\rho$ is a positive constant.

It is easily seen that for $\rho>0$,

$$
f_{n+1}(\rho)>f_{n}(\rho), n=1,2, \ldots
$$

Observe that when $\rho>1 / e$ then

$$
\lim _{n \rightarrow \infty} f_{n}(\rho)=+\infty
$$

because otherwise the sequence $\left\{f_{n}(\rho)\right\}$ would have a finite limit $f_{0}$, such that

$$
f_{0}=e^{\rho f_{0}} .
$$

Using the known ineqality $e^{x} \geq e x$, we have

$$
f_{0}=e^{\rho f_{0}} \geq e \rho f_{0}>f_{0}
$$

which is a contradiction.

When $1 / e<\rho<1$, we also define a sequence $\left\{g_{m}(\rho)\right\}$ by

$$
g_{1}(\rho)=\frac{2(1-\rho)}{\rho^{2}}, \quad g_{m+1}(\rho)=\frac{2(1-\rho)}{\rho^{2}+\frac{2}{g_{m}^{2}(\rho)}}, \quad m=1,2, \ldots
$$

It is easily seen that for $1 / e<\rho<1$

$$
g_{m+1}(\rho)<g_{m}(\rho), m=1,2, \ldots .
$$

We now consider the first order delay differential inequality

$$
x^{\prime}(t)+P(t) x(t-\tau) \leq 0
$$

and the first order advanced differential inequality

$$
x^{\prime}(t)-P(t) x(t+\tau) \geq 0
$$

In order to prove our main results, we present several useful lemmas. 
Lemma 1. Suppose that (3) holds and let $x(t)$ be a solution of inequality (6) on $\left[t_{x}, \infty\right)$, where $t_{x} \geq t_{0}$. Further assume that there exist $t_{1}\left(t_{1} \geq t_{x}\right)$ and positive constant $\rho$ such that

$$
\int_{t-\tau}^{t} P(s) d s \geq \rho>\frac{1}{e}, \quad \text { for } t \geq t_{1}
$$

and that there exist $T_{0} \geq t_{1}$ and $T \geq T_{0}+3 \tau$ such that $x(t)$ is positive on $T_{0} \leq t \leq T$. Then for some $n \geq 1$

$$
\frac{x(t-\tau)}{\sim_{(t)}^{( \pm)}}>f_{n}(\rho), \quad \text { for } t \in\left[T_{0}+(2+n) \tau, T\right]
$$

where $f_{n}(\rho)$ is defined by (4).

Proof: Let $x(t)$ be a positive solution of inequality (6) on $\left[T_{0}, T\right]$. Then by (6) we get

$$
x^{\prime}(t) \leq-P(t) x(t-\tau) \leq 0, \quad \text { for } t \in\left[T_{0}+\tau, T\right]
$$

which implies that $x(t)$ is nonincreasing on $\left[T_{0}+\tau, T\right]$. It follows that

$$
\frac{x(t-\tau)}{x(t)} \geq 1, \quad \text { for } t \in\left[T_{0}+2 \tau, T\right] .
$$

When $T_{0}+3 \tau \leq t \leq T$, dividing (6) by $x(t)$, and integrating from $t-\tau$ to $t$, we get

$$
\ln \left(\frac{x(t)}{x(t-\tau)}\right)+\int_{t-\tau}^{t} P(s) \frac{x(s-\tau)}{x(s)} d s \leq 0 .
$$

By using (8) and (11), we have

$$
\ln \left(\frac{x(t-\tau)}{x(t)}\right) \geq \int_{t-\tau}^{t} P(s) \frac{x(s-\tau)}{x(s)} d s \geq \rho
$$

It follows that

$$
\frac{x(t-\tau)}{x(t)} \geq e^{\rho}=f_{1}(\rho), \quad \text { for } t \in\left[T_{0}+3 \tau, T\right]
$$

Repeating the above procedure, we get

$$
\frac{x(t-\tau)}{x(t)} \geq e^{\rho f_{n-1}(\rho)}=f_{n}(\rho), \quad \text { for } t \in\left[T_{0}+(2+n) \tau, T\right] .
$$


The proof of Lemma 1 is completed.

Lemma 2. Suppose that (3) holds and let $x(t)$ be a solution of inequality (6) on $\left[t_{x}, \infty\right)$, where $t_{x} \geq t_{0}$. Further assume that there exist $t_{1}\left(t_{1} \geq t_{x}\right)$ and positive constant $\rho<1$ such that

$$
\int_{t-\tau}^{t} P(s) d s \geq \rho>\frac{1}{e}, \quad \text { for } t \geq t_{1}
$$

and that there exist $T_{0} \geq t_{1}$ and a positive integer $N \geq 2$ such that $x(t)$ is positive on $T_{0} \leq t \leq T_{0}+(2+N) \tau$. Then for some $m \leq N-1$

$$
\frac{x(t-\tau)}{x(t)}<g_{m}(\rho), \quad \text { for } t \in\left[T_{0}+3 \tau, T_{0}+(2+N-m) \tau\right]
$$

where $g_{m}(\rho)$ is defined by (5).

Proof: Let $x(t)$ is a positive solution of inequality (6) on $\left[T_{0}, T_{0}+(2+N) \tau\right]$. From (14), when $t \geq T_{x}$ we know that

$$
\int_{t-\tau}^{t} P(s) d s \geq \rho \text { and } \int_{t}^{t+\tau} P(s) d s \geq \rho .
$$

Observe that $f(\lambda)=\int_{t}^{\lambda} P(s) d s$ is a continuous function, $f(t)=0$ and $f(t+\tau) \geq \rho$ and there exists $\lambda_{t}$ such that $\int_{t}^{\lambda_{t}} q(s) d s=\rho$, where $t<\lambda_{t}<t+\tau$.

When $T_{0}+3 \tau \leq t \leq T_{0}+(2+N-1) \tau$, integrating both sides of (6) from $t$ to $\lambda_{t}$, we obtain

$$
x(t)-x\left(\lambda_{t}\right) \geq \int_{t}^{\lambda_{t}} P(s) x(s-\tau) d s .
$$

Since $t \leq s \leq t+\tau$, we easily see that $T_{0}+2 \tau \leq t-\tau \leq s-\tau \leq t$. Integrating both side of (6) from $s-\tau$ to $t$, we get

$$
x(s-\tau)-x(t) \geq \int_{s-\tau}^{t} P(u) x(u-\tau) d u .
$$

From (10), it is clear that $x(u-\tau)$ is nonincreasing on $T_{0}+2 \tau \leq s-\tau \leq u \leq t$, thus, we have

$$
\begin{aligned}
x(s-\tau) & \geq x(t)+x(t-\tau) \int_{s-\tau}^{t} P(u) d u \\
& =x(t)+x(t-\tau)\left\{\int_{s-\tau}^{s} P(u) d u-\int_{t}^{s} P(u) d u\right\} \\
& \geq x(t)+x(t-\tau)\left\{\rho-\int_{t}^{s} P(u) d u\right\}
\end{aligned}
$$


From (16) and (17), we have

$$
\begin{aligned}
x(t) & \geq x\left(\lambda_{t}\right)+\int_{t}^{\lambda_{t}} P(s) x(s-\tau) d s \\
& \geq x\left(\lambda_{t}\right)+\int_{t}^{\lambda_{t}} P(s)\left\{x(t)+x(t-\tau)\left(\rho-\int_{t}^{s} P(u) d u\right)\right\} d s \\
& =x\left(\lambda_{t}\right)+\rho x(t)+\rho^{2} x(t-\tau)-x(t-\tau) \int_{t}^{\lambda_{t}} d s \int_{t}^{s} P(s) P(u) d u .
\end{aligned}
$$

As is well known, the identical relation

$$
\int_{t}^{\dot{n}_{t}} d s \int_{t}^{\circ} P(s) P(u) d u=\int_{t}^{\dot{\lambda}_{t}} d u \int_{u}^{\dot{n}_{t}} P(s) P(u) d s
$$

holds. On the right hand side we exchange the variables of integration $s$ and $u$. The above equality becomes

$$
\int_{t}^{\lambda_{t}} d s \int_{t}^{s} P(s) P(u) d u=\int_{t}^{\lambda_{t}} d s \int_{s}^{\lambda_{t}} P(u) P(s) d u
$$

which implies

$$
\begin{aligned}
\int_{t}^{\lambda_{t}} d s \int_{t}^{s} P(s) P(u) d u & =\frac{1}{2} \int_{t}^{\lambda_{t}} d s \int_{t}^{\lambda_{t}} P(u) P(s) d u \\
& =\frac{1}{2}\left(\int_{t}^{\lambda_{t}} P(s) d s\right)^{2}=\frac{\rho^{2}}{2}
\end{aligned}
$$

Substituing this into (18), we have

$$
x(t) \geq x\left(\lambda_{t}\right)+\rho x(t)+\frac{\rho^{2}}{2} x(t-\tau) .
$$

Noting that

$$
x\left(\lambda_{t}\right)>0, \quad \text { for } t \in\left[T_{0}+3 \tau, T_{0}+(2+N-1) \tau\right]
$$

by (19), we get

$$
\frac{x(t-\tau)}{x(t)}<\frac{2(1-\rho)}{\rho^{2}}=g_{1}(\rho), \quad \text { for } t \in\left[T_{0}+3 \tau, T_{0}+(2+N-1) \tau\right]
$$

When $T_{0}+3 \tau \leq t \leq T_{0}+(2+N-2) \tau$, we easily see that $T_{0}+3 \tau \leq t \leq \lambda_{t} \leq t+\tau \leq$ $T_{0}+(2+N-1) \tau$. 
Thus, by (20), we have

$$
x\left(\lambda_{t}\right)>\frac{1}{g_{1}(\rho)} x\left(\lambda_{t}-\tau\right), \quad \text { for } t \in\left[T_{0}+3 \tau, T_{0}+(2+N-2) \tau\right] .
$$

Since $x(t)$ is nonincreasing on $\left[T_{0}+\tau, T_{0}+(2+N) \tau\right]$ and $T_{0}+2 \tau \leq \lambda_{t}-\tau<t<\lambda_{t}<$ $T_{0}+(2+N-1) \tau$, we get

$$
x\left(\lambda_{t}\right)>\frac{1}{g_{1}(\rho)} x\left(\lambda_{t}-\tau\right) \geq \frac{1}{g_{1}(\rho)} x(t)>\frac{1}{g_{1}^{2}(\rho)} x(t-\tau) .
$$

Substituting this into (19), we have

$$
x(t)>\frac{1}{g_{1}^{2}(\rho)} x(t-\tau)+\rho x(t)+\frac{\rho^{2}}{2} x(t-\tau), \quad \text { for } t \in\left[T_{0}+3 \tau, T_{0}+(2+N-2) \tau\right]
$$

Therefore

$$
\frac{x(t-\tau)}{x(t)}<\frac{2(1-\rho)}{\rho^{2}+\frac{2}{g_{1}^{2}(\rho)}}=g_{2}(\rho), \quad \text { for } t \in\left[T_{0}+3 \tau, T_{0}+(2+N-2) \tau\right] .
$$

Repeating the above procedure, we obtain

$$
\frac{x(t-\tau)}{x(t)}<\frac{2(1-\rho)}{\rho^{2}+\frac{2}{g_{m-1}^{2}(\rho)}}=g_{m}(\rho), \quad \text { for } t \in\left[T_{0}+3 \tau, T_{0}+(2+N-m) \tau\right]
$$

The proof of Lemma 2 is completed.

The above lemmas can be extended to the inequality (7). Since the proofs are similar we omit them and we formulate only the corresponding results.

LEMmA 3. Suppose that (3) holds and let $x(t)$ be a solution of inequality (7) on $\left[t_{x}, \infty\right)$, where $t_{x} \geq t_{0}$. Further assume that there exist $t_{1}\left(t_{1} \geq t_{x}\right)$ and positive constant $\rho$ such that

$$
\int_{t}^{t+\tau} P(s) d s \geq \rho>\frac{1}{e}, \quad \text { for } t \geq t_{1}
$$

and that there exist $T_{0} \geq t_{1}$ and a positive integer $N \geq 2$ such that $x(t)$ is positive on $T_{0} \leq t \leq T_{0}+(2+N) \tau$. Then for some $n \leq N$

$$
\frac{x(t+\tau)}{x(t)} \geq f_{n}(\rho), \quad \text { for } t \in\left[T_{0}, T_{0}+(N-n) \tau\right]
$$


where $f_{n}(\rho)$ is defined by (4).

LEMMA 4. Suppose that (3) holds and let $x(t)$ be a solution of inequality (7) on $\left[t_{x}, \infty\right)$, where $t_{x} \geq t_{0}$. Further assume that there exist $t_{1}\left(t_{1} \geq t_{x}\right)$ and positive constant $\rho<1$ such that

$$
\int_{t}^{t+\tau} P(s) d s \geq \rho>\frac{1}{e}, \quad \text { for } t \geq t_{1}
$$

and that there exist $T_{0} \geq t_{1}$ and $T \geq T_{0}+4 \tau$ such that $x(t)$ is positive on $T_{0} \leq t \leq T$. Then for $t \in\left[T_{0}+m \tau, T-3 \tau\right]$

$$
\frac{x(t+\tau)}{x(t)}<g_{m}(\rho)
$$

where $m$ is a positive integer and $g_{m}(\rho)$ is defined by (5).

\section{Main Results}

In this section we shall apply the lemmas in section 2 to establish estimates for the distance between adjacent zeros of the solutions of equations (1) and (2).

In the following, $d(x)$ denotes the distance between adjacent zeros of the solution $x(t)$ of $(1)$ or $(2)$.

THEOREM 1. Suppose that (3) holds and let $x(t)$ be a solution of $(1)$ on $\left[t_{x}, \infty\right)$, where $t_{x} \geq t_{0}$. Further assume that there exist $t_{1}\left(t_{1} \geq t_{x}\right)$ and positive constant $\rho$ such that

$$
\int_{t-\tau}^{t} P(s) d s \geq \rho>\frac{1}{e}, \quad \text { for } t \geq t_{1}
$$

Then $x(t)$ on $\left[t_{1}, \infty\right)$ has arbitrarily large zeros and $d(x)<\left(2+k_{\rho}\right) \tau$, where

$$
k_{\rho}= \begin{cases}1, & \text { when } \rho \geq 1, \\ \min _{n \geq 1, m \geq 1}\left\{n+m \mid f_{n}(\rho) \geq g_{m}(\rho)\right\}, & \text { when } 1 / e<\rho<1\end{cases}
$$

and $f_{n}(\rho), g_{m}(\rho)$ are defined by (4) and (5).

Proof: It suffices to prove that for any $T_{0} \geq t_{1}$ the solution $x(t)$ of $(1)$ has zeros on $\left[T_{0}, T_{0}+k_{\rho} \tau\right]$. Otherwise, without loss of generality, we assume that $x(t)$ is positive on $\left[T_{0}, T_{0}+k_{\rho} \tau\right]$. We consider the following two cases: 
CASE 1. $\rho \geq 1$

In this case, $k_{\rho}=1$. From (1) and (3) we get

$$
x^{\prime}(t)=-P(t) x(t-\tau) \leq 0, \quad \text { for } t \in\left[T_{0}+\tau, T_{0}+3 \tau\right]
$$

which implies that $x(t)$ is nonincreasing on $\left[T_{0}+\tau, T_{0}+3 \tau\right]$, and thus

$$
x(t) \geq x\left(T_{0}+2 \tau\right), \quad \text { for } t \in\left[T_{0}+\tau, T_{0}+2 \tau\right] .
$$

Integrating both sides of (1) from $T_{0}+2 \tau$ to $T_{0}+3 \tau$, we obtain

$$
\begin{aligned}
x\left(T_{0}+3 \tau\right) & =x\left(T_{0}+2 \tau\right)-\int_{T_{0}+2 \tau}^{T_{0}+3 \tau} P(s) x(s-\tau) d s \\
& \leq x\left(T_{0}+2 \tau\right)\left\{1-\int_{T_{0}+2 \tau}^{T_{0}+3 \tau} P(s) d s\right\} .
\end{aligned}
$$

By (27) and (29), we have

$$
x\left(T_{0}+3 \tau\right) \leq x\left(T_{0}+2 \tau\right)(1-\rho) \leq 0
$$

which is a contradiction.

CASE 2. $1 / e<\rho<1$

Without loss of generality, we assume that $x(t)$ is positive on $\left[T_{n}, T_{n}+\left(2+k_{n}\right) \tau\right]$. Let $k_{\rho}=n^{*}+m^{*}$ where

$$
f_{n^{*}}(\rho) \geq g_{m^{*}}(\rho)
$$

By Lemma 1, we have

$$
\frac{x(t-\tau)}{x(t)} \geq f_{n^{*}}(\rho), \quad \text { for } t \in\left[T_{0}+\left(2+n^{*}\right) \tau, T_{0}+\left(2+k_{\rho}\right) \tau\right]
$$

On the other hand, by Lemma 2 we get

$$
\frac{x(t-\tau)}{x(t)}<g_{m^{*}}(\rho), \quad \text { for } t \in\left[T_{0}+3 \tau, T_{0}+\left(2+k_{\rho}-m^{*}\right) \tau\right] .
$$

Setting $t=T_{0}+\left(2+n^{*}\right) \tau$ in (30) and (31), we obtain

$$
f_{n^{*}}(\rho) \leq \frac{x\left(T_{0}+\left(1+n^{*}\right) \tau\right)}{x\left(T_{0}+\left(2+n^{*}\right) \tau\right)}<g_{m^{*}}(\rho)
$$

which contradicts (30) and completes the proof. 
REMARK 1. Theorem 1 improves $[1$, Theorem 2.2.1 and 2.2.2] [4, Theorem 1 and 2] and $[5$, Theorem 1].

Corollary 1. When $P(t)$ in (1) is a positive constant $p, p \tau=\rho>1 / e$, and $x(t)$ is a solution of $(1)$ on $\left[t_{x}, \infty\right)$, then $x(t)$ on $\left[t_{x}, \infty\right)$ has arbitrarily large zeros and $d(x)<\left(2+k_{\rho}\right) \tau$, where $k_{\rho}$ is defined by $(28)$.

Using a similar method to the proof of Theorem 1, by Lemmas 3 and 4, we obtain:

THEOREM 2. Assume that (3) holds and $x(t)$ is a solution of $(2)$ on $\left[t_{x}, \infty\right)$, where $t_{x} \geq t_{0}$. Assume that there exist $t_{1}\left(t_{1} \geq t_{x}\right)$ and positive constant $\rho$ such that

$$
\int_{t}^{t+\tau} P(s) d s \geq \rho>\frac{1}{e}, \quad \text { for } t \geq t_{1} .
$$

Then $x(t)$ on $\left[t_{1}, \infty\right)$ has arbitrarily large zeros and $d(x)<\left(2+k_{\rho}\right) \tau$, where $k_{p}$ is defined by (28).

Corollary 2. When $P(t)$ in (2) is a positive constant $p, p \tau=\rho>1 / e$, and $x(t)$ is a solution of $(2)$ on $\left[t_{x}, \infty\right)$, then $x(t)$ on $\left[t_{x}, \infty\right)$ has arbitrarily large zeros and $d(x)<\left(2+k_{\rho}\right) \tau$, where $k_{\rho}$ is defined by $(28)$.

EXAMPLE 1. Consider the delay differential equation

$$
x^{\prime}(t)+x(t-0.4)=0
$$

where $p=1$. We have $\rho=\tau=0.4$ and $f_{1}(\rho)=1.491 \ldots, f_{2}(\rho)=1.816 \ldots, f_{3}(\rho)=$ $2.067 \ldots, \ldots, f_{10}(\rho)=4.387 \ldots, f_{11}(\rho)=5.784 \ldots, f_{12}(\rho)=10.111 \ldots, \ldots ; g_{1}(\rho)=$ $7.500 \ldots, g_{2}(\rho)=6.136 \ldots, g_{3}(\rho)=5.631 \ldots, g_{4}(\rho)=5.379 \ldots, \ldots$.

Thus, we find $f_{n}(\rho)<5<g_{m}(\rho), 1 \leq n \leq 10, m \geq 1 ; f_{11}(\rho)>g_{m}(\rho), m \geq 3 ;$ $f_{12}(\rho)>g_{m}(\rho), m \geq 1$.

Hence, by Corollary 1 , we have $k_{\rho}=12+1=13$ and $d(x)<15 \times 0.4$. This improves the result in [4]: $d(x)<28 \times 0.4$.

EXAmple 2. Consider the differential equation

$$
x^{\prime}(t)+x(t-0.55)=0
$$

where $p=1$. We have $\rho=\tau=0.55$ and $f_{1}(\rho)=1.733 \ldots, f_{2}(\rho)=2.594 \ldots, f_{3}(\rho)=$ $4.165 \ldots, f_{4}(\rho)=9.884 \ldots, \ldots g_{1}(\rho)=2.975, g_{2}(\rho)=1.703 \ldots, g_{3}(\rho)=$ $0.907 \ldots, \ldots$

Thus, we find $f_{1}(\rho)>g_{m}(\rho), m \geq 2 ; f_{2}(\rho)>g_{m}(\rho), m \geq 2 ; f_{3}(\rho)>$ $g_{m}(\rho), m \geq 1$. Hence, by Corollary 3.1 , we have $k_{\rho}=1+2=3$ and $d(x)<5 \times 0.55$.

REMARK 2. We can also apply our results to neutral differential equations

$$
\frac{d}{d t}[x(t)+C(t) x(t-r)]+P(t) x(t-\sigma)=0
$$

where $C, P \in C\left(\left[t_{0}, \infty\right), R^{+}\right)$and $r, \sigma \in R^{+}$. We can improve the results in $[6,7,8]$. 


\section{REFERENCES}

[1] L.H. Erbe, Q.K. Kong and B.G. Zhang, Oscillation theory for functional differential equations, Monographs and Textbooks in Pure and Applied Mathematics 190 (Marcel Dekker, Inc., New York, 1995).

[2] K. Gopalsamy, Stability and oscillation in delay differential equations of population dynamics (Kluwer Academic Publishers, Dordrecht, 1992).

[3] I. Györi and G. Ladas, Oscillation theory of differential equations with applications (Clarendon Press, Oxford, 1991).

[4] F.X. Liang, 'The distribution of zeros of solutions of first-order delay differential equations', J. Math. Anal. Appl. 186 (1994), 383-392.

[5] Y. Zhou, 'On the distribution of zeros of solutions of delay differential equations', Ann. Hunan Math. 16 (1996), 108-110.

[6] Y. Zhou, 'The distribution of zeroes of solutions of first order neutral differential equations', Northeast Math. J. 13 (1997), 153-159.

[7] Y. Zhou, 'An estimate for distance between adjacent zeros of solutions of neutral equations', Chinese Quart. J. Math. 11 (1996), 47-52.

[8] Y. Zhou and Z.C. Wang, 'The distribution of zeroes of solutions of neutral equations', Appl. Math. Mechanics 18 (1997), 1197-1204.

Department of Mathematics

Xiangtan University

Hunan 411105

People's Republic of China 